\section{Ultrafast Laser Radiation Generated Silicon Nanocrystals Embedded into a Glass Matrix}

The formation of photo-active Si nanocrystals deeply embedded in the bulk is not a trivial matter as most techniques form such nanocrystals only on the surface or shallowly embedded into the bulk. A.H. Nejadmalayeri and Coworkers from the University of Toronto, Canada, together with J. Burghoff and coworkers from the Friedrich-SchillerUniversität, Jena and J. Kaspar from the Fraunhofer-Institut für Werkstoff und Strahltechnik in Dresden, Germany, reported in the December 15, 2007 issue of Optics Letters (p. 3474) the laser generation of Si nanocrystals within bulk transparent media deep below the surface.

The researchers used linearly polarized mid-infrared ultrafast radiation at a wavelength of $2400 \mathrm{~nm}$ to propagate in the transparency window of Si. They focused the beam into Si wafers overcoated with 20- $\mu \mathrm{m}$ thick silicon oxide, and scanned the samples transversely using a motorized translation stage. The laser exposure created damage tracks along the [100] crystallographic direction at the $\mathrm{Si} / \mathrm{SiO}_{2}$ interface, leaving the top oxide surface intact, and generated optical waveguides for $1300 \mathrm{~nm}$ and $1550 \mathrm{~nm}$ radiation with moderate losses of $\sim 1 \mathrm{~dB} / \mathrm{cm}$. Transmission electron micrographs and diffraction patterns showed small crystallites, 5-30 nm in size, without any particular order within the amorphous oxide immediately above the silicon-glass interface.

"Although $\mathrm{Si}$ and $\mathrm{SiO}_{2}$ are transparent to the laser radiation, the interface exhibits relatively low damage threshold due to impact ionization and/or other nonlinear interactions, that cause a rapid heating and melting of a thin Si layer at the interface, followed by the melting of the adjacent oxide. Upon cooling, the molten glass resolidifies, and the silicon droplets recrystallize, forming a heterogeneous mixture of $\mathrm{Si}$ nanoparticles randomly oriented in the glass," the researchers said. The Si matrix close to the modified region was disordered and under planar compressive stress, as confirmed by Raman characterization. According to the researchers, this stress caused a density change that can induce an increase of refractive index, responsible for the optical waveguiding observed.

However, the researchers still do not know if this phenomenon is a unique characteristic of the ultrashort pulsed laser interaction with the $\mathrm{Si} / \mathrm{SiO}_{2}$ system or if it can occur in other systems like $\mathrm{Si} / \mathrm{Si}_{3} \mathrm{~N}_{4}$. The researchers consider the close proximity of the nanocrystals to the evanescent fields of the guided modes that were observed in these structures "very interesting." They said, "Considering the attractive luminescence properties, their proximity to such guided wave optics in a single step process suggests new opportunities to explore means of making compact Si-based light sources."

JOAN J. CARVAJAL

\section{Boron-Doped Titanium Nitride Nanocomposite Thin Films Exhibit Broadband Photoluminescence}

Titanium nitride (TiN) has been used as a template to grow gallium nitride $(\mathrm{GaN})$ thin films due to its high electrical conductivity and small lattice mismatch with GaN. TiN films are usually doped with boron to increase their hardness. However, the photoluminescence (PL) properties of TiN-based thin films have rarely been reported. S.-G. Lu, who is affiliated with City University of Hong Kong and Central South University, China, Z.-K. Xu from City University of Hong Kong, Y.-H. Lu from Beijing University of Science and Technology, and K.-W. Cheah from Hong Kong Baptist University have measured the photoluminescence properties of boron-doped TiN thin films with nanosized grains as a function of $\mathrm{B}$ content and temperature.

As reported in the December 2007 issue of the Journal of the American Ceramic Society (p. 4002; DOI: 10.111/j.1551-2916.2007. 02040.x), the researchers prepared a series of TiBN thin films with different compositions using a reactive close-field unbalanced magnetron sputtering (RCFUMS) system at room temperature. The films were all $\sim 1.5 \mu \mathrm{m}$ thick and deposited on polished (100) silicon substrates with a 40-50-nm thick Ti buffer layer to improve adhesion and reduce stress.

After the preparation, the researchers characterized the composition and structure of the thin films and investigated their photoluminescence. X-ray diffraction (XRD) results reveal that the grain size in the films decreases and the diffraction peaks of each pattern more or less shift toward low $2 \theta$ angles with increasing B content. But all the XRD peaks of TiBN with the highest amount of B studied disappear due to the tiny grain size, although weak diffraction patterns are observed by transmission electron microscopy (TEM). The PL spectra of samples at different temperatures show that at low temperature (12 K), each PL spectrum has two main PL peaks. The strong peak at $3.20 \mathrm{eV}$ is attributed to the recombination between electrons bound to $\mathrm{N}$ vacancies and holes in the valence band, and the weaker peak at $1.63 \mathrm{eV}$ is caused by the recombination of electrons in defects at surface states with holes in the valence band. The PL spectra at high temperatures of the sample with the highest $\mathrm{B}$ content show that a new peak appears near $2.38 \mathrm{eV}$ and blue shifts slightly with increasing temperature. The researchers attributed this PL peak to deep-trap defect emission. The researchers concluded that the change of color from violet to green with temperature may be useful for applications such as optical display devices.

ZHAOYONG SUN

\section{Inorganic-Organic Composite Scaffolds Offer New Material for Tissue Engineering}

Challenges in tissue engineering require the development of materials systems that provide a temporary scaffold mimicking the three-dimensional (3D) natural extracellular matrix. Besides bioactivity, this scaffold must consist of 3D interconnected pore networks with pore size greater than $100 \mu \mathrm{m}$ (for giant pores), thus promoting cell penetration and growth. Inorganic mesoporous bioactive glasses (MBGs) have been developed to meet these requirements. However, MBGs are too brittle to use alone as bone scaffolds. H.-S. Yun of the Korea Institute of Materials Science, S.-J. Heo of Inje University, and their colleagues overcome this problem by developing an inorganic-organic composite material based on MBGs and poly $\varepsilon$-caprolactone (PCL).

As described in the December 25, 2007 issue of Chemistry of Materials (p. 6363; DOI: $10.1021 / \mathrm{cm} 7023923)$, the researchers fabricated hierarchically a 3D porous MBGpolymer composite using a combination of sol-gel, polymer templating, and rapid prototyping (RP) techniques with a robotic deposition device. A virtual 3D scaffold model was first created by computer-aided design and then used to guide the scaffold fabrication from direct extrusion of a MBG-PCL gel paste onto a cooled substrate. The substrate was maintained at low temperature in order to facilitate the solidification of the PCL. At the same time, the researchers designed a heat-controlled blowing system to accelerate the solvent evaporation and thus maintain the 3D scaffold morphology. The researchers reported that they can also control the ease of 3D scaffold formation by adjusting the MBG content of the paste. By applying these techniques, a scaffold with excellent molding capabilities and mechanical properties was obtained, they said.

The researchers evaluated the boneforming activity of the MBG-PCL scaffold in simulated body fluid. Field emission scanning electron microscopy (FE-SEM) was used to monitor the apatite formation 
process. Apatite-like nanoparticles were observed on the MBG-PCL surface after soaking for $4 \mathrm{~h}$, and soaking for $24 \mathrm{~h}$ resulted in a MBG-PCL fully covered with apatite. Likewise, energy dispersive x-ray (EDX) analysis gave the same result. The biocompatibility of the MBG-PCL was evaluated by MTT [3-(4,5-Dimethylthiazol-z-YI)-2,5-Diphenyltetrazolium Bromide] assay. The cell viability on the MBG-PCL surface was found to be significantly larger than that on the PCL surface. These studies demonstrate excellent bioactivity and biocompatibility of this MBGPCL scaffold, the researchers said.

The method developed in this work was used to fabricate a scaffold for tissue engineering. However, by utilizing alternative materials, the researchers showed that this method can be used in "applications involving biomedical devices, drug delivery systems, filters, catalysis, and optics."

CHANG ZHONG

\section{Atomic Force Microscopy Used to Detect Cancer Cells}

Immunohistochemical and cytomorphological analyses are currently used to detect cancer cells, but morphological overlap between tumor and normal cell types often poses problems for these techniques. In the past few years, however, an increase in cell elasticity has been recognized as a marker for disease and associated with cell adhesion and cytoskeletal organization. S.E. Cross and J.K. Gimzewski at the California NanoSystems Institute and the University of California, Los Angeles, and Y.-S. Jin and J. Rao at the University of California, Los Angeles used atomic force microscopy (AFM) to distinguish cancerous cells from normal cells.

As reported in a letter published in the December 2, 2007 issue of Nature Nanotechnology (p. 780; DOI:10.1038/nnano. 2007.388), the researchers used standard protocols to collect from patients with suspected metastatic adenocarcinoma samples of malignant and benign mesothelial cells in pleural effusions (adenocarcinoma is a cancer that originates in glandular tissue; mesothelial cells form part of the membranes covering body cavities, and an effusion is an abnormally large collection of fluid in the space surrounding the lungs). Ex vivo growth during a 12-h incubation period differentiated normal cells, which have a large, flat morphology, from benign cells, which exhibit anchorage-resistant morphology such as rounding. For each cell, elasticity was quantified as Young's modulus $E$ from force-displacement curves recorded from AFM performed at a rate of $1 \mathrm{~Hz}$ at $37^{\circ} \mathrm{C}$. Using samples from seven patients, average $E$ values of $0.53 \pm$
$0.10 \mathrm{kPa}$ and $1.97 \pm 0.70 \mathrm{kPa}$ were obtained for 40 malignant and 48 benign mesothelial cells, respectively. Similar average $E$ values were found for samples from a single patient, showing that the cell stiffness of metastatic cancer cells is about $73 \pm 11 \%$ less than benign mesothelial cells. Malignant cells displayed a narrow distribution of $E$ values while the benign cells displayed a broad peak.

For one particular clinical sample consisting of cells that were difficult to classify as either benign or malignant, ex vivo culturing resulted in two populations of cells with different morphologies. However, nanomechanical analysis based on AFM measurements determined that both populations were malignant cells, which was confirmed with immunohistochemical staining. The researchers said that "the correlation of cytomechanical measurements with immunohistochemical analysis suggests that nanomechanical measurements of cancer cells has potential for the detection of cancer and may aid in personalized selection of medication and drug screening, especially in body cavity effusions where accurate diagnosis based solely on morphology has to date been challenging." STEVEN TROHALAKI

\section{Embedded Capacitive Circuit Detects, Characterizes, and Manipulates Droplets in Microfluidic Systems}

Droplets in microfluidic systems are presently detected using optical devices, such as single element photodiodes for counting or high-speed charge-coupled device image sensors with imageprocessing software for imaging. A group of researchers from The Hong Kong University of Science and Technology reported in the October-December 2007 issue of Biomicrofluidics (DOI: $10.1063 / 1.2795392)$ on a novel method for real-time detecting, characterizing, and controlling of such droplets through the use of capacitive sensors; the dielectric constant contrast between the fluid of interest and the carrying fluid is the only prerequisite for the method functionality.

The research group, including $X$. Niu, M. Zhang, S. Peng, W. Wen, and P. Sheng, designed a microfluidic chip that embeds from the fabrication process (using soft lithography) a series of parallel electrodes placed across the microfluidic channel, some of which are connected to a capacitance resonance detection system and others to a square wave signal generator for in situ manipulation of the droplets.

The researchers were able to calibrate the device for measurements involving length (and subsequently volume) of nano- and picoliter range aqueous droplets in silicone oil by analyzing the resonance circuit voltage changes as the droplets were passing through the electrodes. Very good accuracy was obtained for droplet lengths bigger than half of the electrode width, and a detection frequency of more than $1 \mathrm{kHz}$ (1100 droplets per second) has been attained, limited in this case only by the sustainable pressure of the chip and not by the detection system. The droplets velocity was also easily calculated by measuring the time required to reach from one set of electrodes to the other.

The system was subsequently calibrated and employed to detect droplets of different composition (ethylene glycol and, respectively, water) and to sort them in two different branches of a T-shaped microchannel using a feedback system that employs a set of electrodes with a positive high potential to charge the droplets and another set that can preferentially change polarity to direct the droplets through either of the branches. The delay time of the feedback system was adjusted for the droplet's velocity so that a perfect match was obtained between detection and manipulation.

The researchers demonstrated that their method of real-time labeling, sorting, and manipulation of droplets can provide a very simple and cost-effective alternative for portable devices involved in biomicrofluidic processing, digital microfluidics, microchemical reactions, and environmental monitoring.

EUgEN PANAITESCU

\section{Quasi-Forbidden Bragg Peaks Demonstrated as Inherent Property in Homogeneous Ordered Soft Materials}

Bragg peaks exist in highly ordered soft materials that cannot be indexed assuming homogeneous crystal structures and that have been attributed to changes in the crystal structure induced by the ordering process. S. Förster and co-workers from the University of Hamburg, Germany, together with S.V. Roth from DESY, Germany and P. Lindner from the Institute Laue-Langevin, France, have demonstrated that these unexpected Bragg peaks are an inherent property of homogeneous ordered soft materials and arise from the spontaneous ordering allowed by the weak interaction potential that tolerates imperfections of the constituent structures that limit the coherence of the crystalline lattice. Their results have been reported in the November 2007 issue of Nature Materials (p. 888; DOI: 10.1038/ nmat1995). "This explained the presence of quasi-forbidden Bragg peaks in lyotrop- 